\title{
Latest Results of the STEREO Sterile Neutrino Search at the ILL Grenoble
}

\author{
Aurélie Bonhomme ${ }^{* \dagger}$ \\ Max-Planck-Institut für Kernphysik \\ E-mail: aurelie.bonhomme@mpi-hd.mpg.de
}

\begin{abstract}
The standard three-family model describing the neutrino oscillation phenomenon is challenged by several experimental anomalies, such as the Reactor Antineutrino Anomaly (RAA), that can be solved by introducing a light sterile neutrino at the eV scale. STEREO probes the indicated oscillation phase-space region using a Gadolinium-doped liquid scintillator segmented neutrino target at 10 meters distance from the compact core of the ILL research reactor, in Grenoble (France). The hypothesis of an oscillation towards a light sterile neutrino is tested by performing a relative comparison of measured $\bar{v}_{e}$ spectra between cells. The data recorded during 119 (211) days of reactor turned on (off) are compatible with the null oscillation hypothesis and reject the original best-fit of the RAA at $99 \%$ C.L.
\end{abstract}

XXIX International Symposium on Lepton Photon Interactions at High Energies - LeptonPhoton2019 August 5-10, 2019

Toronto, Canada

\footnotetext{
* Speaker.

${ }^{\dagger}$ On behalf of the STEREO collaboration
} 


\section{Introduction}

The Reactor Antineutrino Anomaly (RAA) was established in 2011, when a new spectral prediction of reactor antineutrinos showed a $6 \%$ deficit in the rates observed by previous reactor experiments [1]. A possible explanation for this phenomenon introduces sterile neutrinos at the $\mathrm{eV}$ mass-scale into which electron antineutrinos would oscillate. In the simplest model with one sterile neutrino, the probability of disappearance of $\bar{v}_{e}$ at very short baseline is, to a good approximation:

$$
\mathrm{P}_{\bar{v}_{e} \rightarrow \bar{v}_{e}}\left(\mathrm{~L}, \mathrm{E}_{\bar{v}_{e}}\right) \simeq 1-\sin ^{2}\left(2 \theta_{\text {new }}\right) \sin ^{2}\left(1.27 \Delta m_{\text {new }}^{2} \mathrm{~L} / \mathrm{E}_{\bar{v}_{e}}\right)
$$

where $\mathrm{E}_{\bar{v}_{e}}$ is the $\bar{v}_{e}$ energy (in MeV), $\mathrm{L}$ is the distance (in meters) between the core and the detector, and $\theta_{\text {new }}$ and $\Delta m_{\text {new }}^{2}$ the mixing angle and mass splitting introduced by the additional sterile neutrino. The original RAA best-fit parameters are $\left(\sin ^{2}\left(2 \theta_{\text {new }}\right), \Delta \mathrm{m}_{\text {new }}^{2}\right)_{\mathrm{RAA}}=\left(0.14,2.4 \mathrm{eV}^{2}\right)$.

Alternatively, an erroneous prediction of the corresponding $\bar{v}_{e}$ flux - due to underestimated systematics, biases in the conversion method or normalization errors - could cause this deficit. Besides the search for sterile neutrinos, a deep understanding of reactor $\bar{v}_{e}$ spectrum is thus crucial. Moreover, in addition to the RAA, discrepancies in shape with respect to prediction are observed by Double Chooz, Daya Bay, RENO and NEOS. These experiments all measure $\bar{v}_{e}$ from commercial reactors. Measurements close to ${ }^{235} \mathrm{U}$-enriched reactor cores provide valuable complementary information and test the hypothesis of anomalies driven by one single isotope.

In this context, STEREO is designed to be sensitive to the favored RAA region and aims at providing a new high-precision reference measurement of the reactor $\bar{v}_{e}$ spectrum for pure ${ }^{235} \mathrm{U}$.

\section{Experimental setup}

The STEREO active volume for $\bar{v}_{e}$ detection (the Target) consists of six identical cells of $37 \mathrm{~cm}$ length, and covers the distance from 9.2 to 11.3 meters from the ILL compact reactor core in Grenoble [2]. The neutrino oscillation hypothesis associated to sterile neutrinos at the eV massscale can be tested by looking for a relative distortion of the $\bar{v}_{e}$ energy spectrum with distance. Antineutrinos are dominantly produced by fission products of the ${ }^{235} \mathrm{U}$ isotope in the $93 \%$ enriched uranium fuel and are detected via the inverse beta decay (IBD) process in the liquid scintillator in the detector: $\bar{v}_{e}+p \rightarrow e^{+}+n$. The signature of this reaction is a coincidence in time between the prompt energy deposit and annihilation of the positron, and the delayed $\gamma$ cascade coming from the neutron capture after its thermalization and diffusion in the medium. Neutron detection efficiency is enhanced by loading the $2 \mathrm{~m}^{3}$ of the Target liquid with Gadolinium, thanks to the high neutron capture cross-section and energy release $(\sim 8 \mathrm{MeV})$ of this element. The mean capture time of a neutron is about $16 \mu$ s in the Target. The optical separation of the cells is ensured by multi-layer walls consisting of acrylic plates and reflective foils ( $3 \mathrm{M}^{\mathrm{TM}}$ Enhanced Specular Reflector). Light from scintillation produced in the liquid is collected by four 8-inch photomultiplier tubes (PMT) on the top of each cell, placed inside an acrylic buffer and immersed into mineral oil for optical contact. The full energy reconstruction of a Target event is enabled by an outer crown - filled with undoped liquid scintillator - retrieving escaping $\gamma$ 's from positron annihilation or the $\gamma$ cascade from neutron capture. A cut view of the STEREO detector is presented in Fig. 1. 


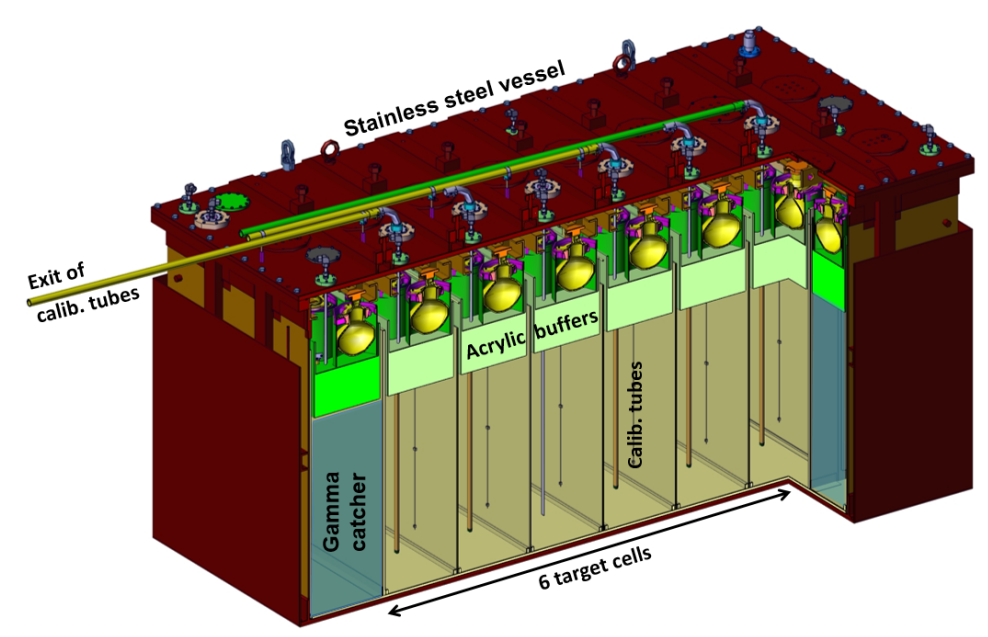

Figure 1: Cut view of the STEREO detector. Six identical Target cells (yellow) are surrounded by the Gamma-Catcher (blue). Acrylics buffers - holding the PMTs - are placed on top of the volumes (green).

The surrounding environment in the reactor building and the detector location at ground level make the measurement challenging. The background is first reduced with passive shielding: the detector is inserted into a support structure holding magnetic shielding, polyethylene, lead and neutron absorbers, with a total mass of about 65 tons. The detector benefits from the protection of a $15 \mathrm{~m}$ water equivalent overburden provided by the water pool around the reactor core, reducing the cosmic background. A Cherenkov muon-veto detector on the top of the STEREO setup acts as an active shielding against this background by tagging muons.

\section{Detector Response}

The response of the liquid and of the electronics are monitored automatically using LEDs with dedicated acquisitions. The energy response of the detector is monitored on a weekly basis using mainly $\gamma$ radioactive sources, while the neutron capture response is studied with a dedicated AmBe neutron source. The Monte-Carlo simulation of the detector is tuned and reproduces energy non-linearities due to quenching effects at the percent level as well as light distributions across cells. Recently, a special attention has been given to the simulation of the $\gamma$ released after the neutron capture on Gadolinium, whose signal is the signature of an IBD interaction. A significant improvement in describing the deexcitation cascades was obtained thanks to the FIFRELIN code, making use of the most up to date experimental data and recommended nuclear models and data evaluations. Fig. 2 shows the improvement with respect to the previously used GLG 4 s im package for the neutron capture spectrum. Further details can be found in [3].

A method taking into account light cross-talk between cells allows to convert the PMT signals into a reconstructed energy. The energy resolution is $9 \%$ at $0.8 \mathrm{MeV}$. Cell-to-cell residual discrepancies between the simulation and the data drive the uncorrelated energy scale uncertainties. The reconstructed energy is monitored in time using the $2.2 \mathrm{MeV} \gamma$ peak from cosmic-induced neutron capture on hydrogen in the liquid. A sub-percent stability - shown in Fig. 3 - is achieved. 


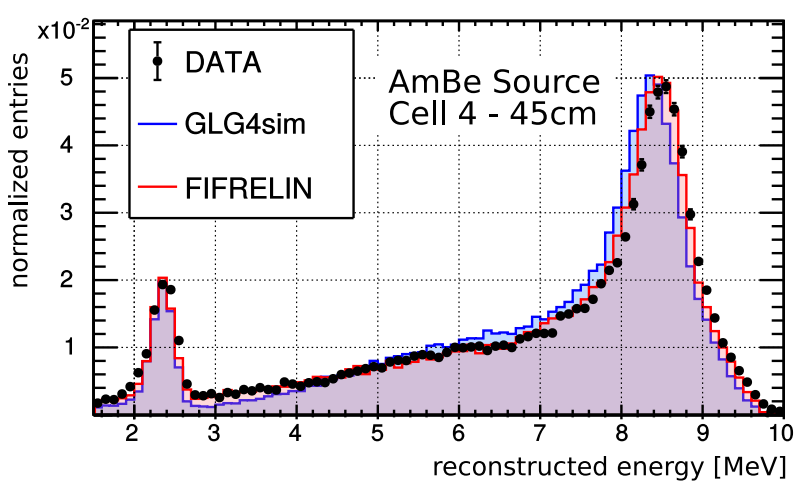

Figure 2: Neutron capture spectrum for an AmBe source deployed in the middle of the fourth Target cell, for data (black) and simulation, with the GLG4 sim (blue) and the FIFRELIN (red) codes .

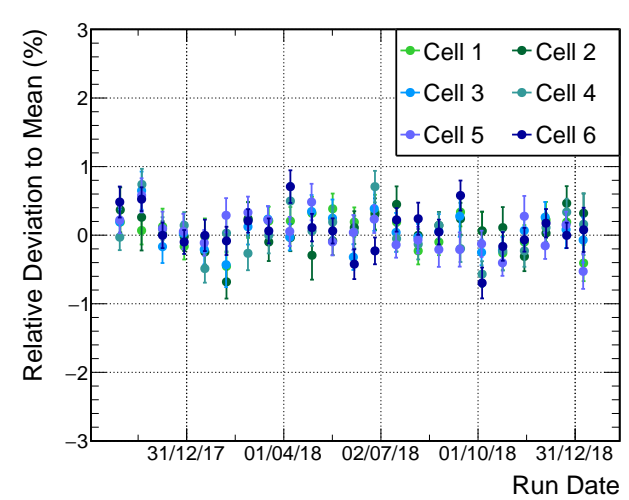

Figure 3: Cell-wise time stability of the $2.2 \mathrm{MeV}$ gamma from neutron captures on hydrogen following cosmic events.

\section{4. $\bar{v}_{e}$ Signal Selection}

The IBD selection for the analysis of Phase-II ${ }^{1}$ presented here was performed on 119 (211) effective days of reactor turned on (off) data, by looking for coincidences between prompt [1.625$7.125 \mathrm{MeV}$ ] and delayed [4.5-10 MeV] signals within a $70 \mu$ s time. In addition to a $100 \mu$ s muonveto, we require no events within a $100 \mu$ s window before and after pair candidates. The latter criteria reduces multi-neutron cascades created by cosmic radiation. Non-tagged muons that stop and decay in the top layer of the detector may be mistaken as IBD candidates. Their asymmetry of charge deposition in the PMTs of the vertex cell is higher than that of events in the detector bulk and was used to reject them. Note that muons stopping deeper in the detector are rejected due to their high energy deposit. The detector segmentation is used to further reduce the IBD background, details are presented in [4]. The Pulse Shape Discrimination capability of the liquid scintillator is based on the different scintillation decay time constants of the liquid depending on the nature of the energy deposit. In STEREO, the relevant discriminating observable is provided by the ratio of the tail charge over the total charge collected from a signal, $\mathrm{Q}_{\text {tail }} / \mathrm{Q}_{\text {tot }}$. A typical distribution is represented in Fig. 4. Theis observable allows to discriminate in the residual correlated background electronic recoils, as in $\bar{v}_{e}$ interactions, from proton recoils, induced by fast neutrons. A linear anticorrelation was observed between the $\mathrm{Q}_{\text {tail }} / \mathrm{Q}_{\text {tot }}$ variable and the liquid temperature. To correct for these drifts, the observable is monitored along time for each energy bin and each cell using the high-statistic sample of single events triggering the detector, and provides a precise correction on a daily basis. Once corrected, the $\mathrm{Q}_{\text {tail }} / \mathrm{Q}_{\text {tot }}$ distributions of the correlated background are studied during the reactor off periods. Induced by cosmic events, the correlated background rate shows a correlation with the atmospheric pressure and the level of the water channel above STEREO. Despite these environmental variations, the shape of the correlated background distributions was shown to be very stable under all the different conditions encountered during data taking. With this reference background shape, the $\bar{v}_{e}$ candidates are directly extracted for each energy bin separately

\footnotetext{
${ }^{1}$ Phase-II stands for data taken between Oct. 2017 and Dec. 2018.
} 
from the simultaneous fit of the $\mathrm{Q}_{\text {tail }} / \mathrm{Q}_{\text {tot }}$ distributions obtained in the reactor-on and reactor-off periods, with only a scaling parameter of the background model, reflecting the local environmental conditions. The accidental component - dominated by electronic recoils - is constrained with accidental pair candidates, obtained by looking for random coincidences in 100 delayed windows for each prompt candidate. A normal distribution is used to model the neutrinos distribution and the neutrino rate in each energy bin and each cell is obtained from the integral estimate of this model. One example of such a fit is shown in Fig. 4.

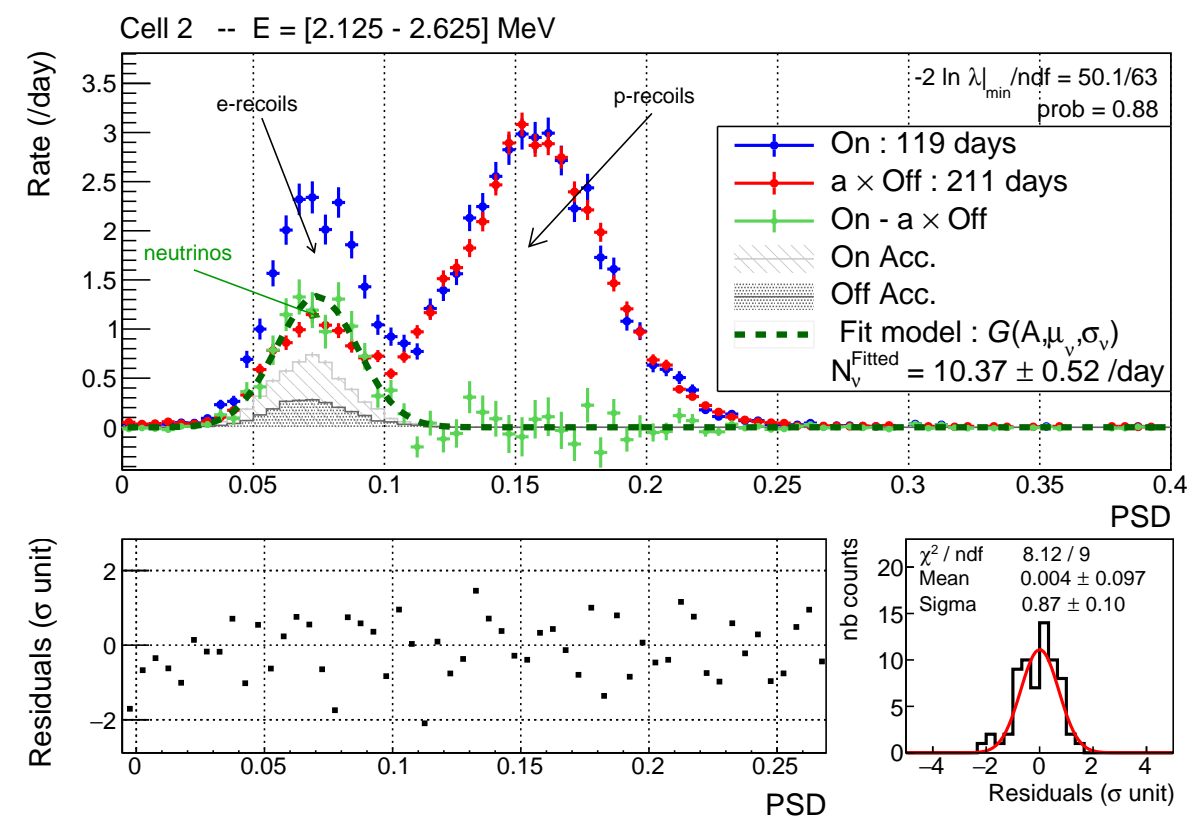

Figure 4: Top frame: example of $\bar{v}_{e}$ rate extraction in one energy bin for cell 2 from the combined fit of the $\mathrm{Q}_{\text {tail }} / \mathrm{Q}_{\text {tot }}$ distributions in reactor-on (blue) and reactor-off (red). The background model from reactor-off is scaled with the free parameter $a$, and the neutrino distribution is adjusted by a Gaussian model (green). The accidental distributions (gray) are estimated from the delayed windows method. Residuals of the global fit are shown in the bottom frames.

\section{Oscillation Analysis}

The oscillation analysis consists of looking for relative distortions of the $\bar{v}_{e}$-spectrum between the 6 cells. The measured spectra $\left(D_{l, i}\right)$ and simulated spectra $\left(M_{l, i}\right)$ are compared using the following $\chi^{2}$ formalism:

$$
\begin{aligned}
\chi^{2}= & \sum_{l}^{\text {Ncells }} \sum_{i}^{N_{E}}\left(\frac{D_{l, i}-\phi_{i} M_{l, i}(\mu, \sigma, \alpha)}{\sigma_{l, i}}\right) \\
& +\sum_{l}^{\text {Ncells }}\left(\frac{\alpha_{l}^{\text {NormU }}}{\sigma_{l}^{\text {NormU }}}\right)^{2}+\sum_{l}^{\text {Ncells }}\left(\frac{\alpha_{l}^{\text {EscaleU }}}{\sigma_{l}^{\text {EscaleU }}}\right)^{2}+\left(\frac{\alpha_{0}^{\text {EscaleC }}}{\sigma_{0}^{\text {EscaleC }}}\right)^{2}
\end{aligned}
$$

where the $\phi_{i}$ are free normalization parameters for each energy bin $i$, common for all cells, allowing an oscillation test independent of a reactor-based prediction. 
All detector response effects (relative efficiencies, non-linearities...) are taken into account in the simulated spectra. This method is insensitive to the spectrum prediction and exploits the relative difference between the cells, thus reducing common systematics. The only remaining systematics are the uncorrelated normalizations ( $\left.\alpha_{l}^{\mathrm{NormU}}\right)$, energy scale discrepancies between cells $\left(\alpha_{l>0}^{\mathrm{EscaleU}}\right)$, and the common energy scale $\left(\alpha_{0}^{\text {EscaleC }}\right)$. They are included in the formalism via nuisance parameters $\alpha$ constrained by pull-terms.

Data are compatible with the null oscillation hypothesis. The corresponding p-value of $40 \%$ is determined by generating a large number of pseudo-experiments. The null hypothesis cannot be rejected and a Raster Scan method is used to produce the exclusion contour at $90 \%$ C.L. shown in Fig. 5. In particular, the original RAA best-fit point [1] is excluded at $99 \%$ C.L.

The STEREO experiment is continuing data taking under very stable conditions, and will cover its full sensitivity to the RAA region doubling the statistics by mid-2020. Meanwhile, STEREO will be able to provide the pure $\bar{v}_{e}$ spectrum associated to the fission of ${ }^{235} \mathrm{U}$ and an absolute measurement of its flux with unprecedented precision, shedding some complementary light on the RAA and the reactor spectral distortions.

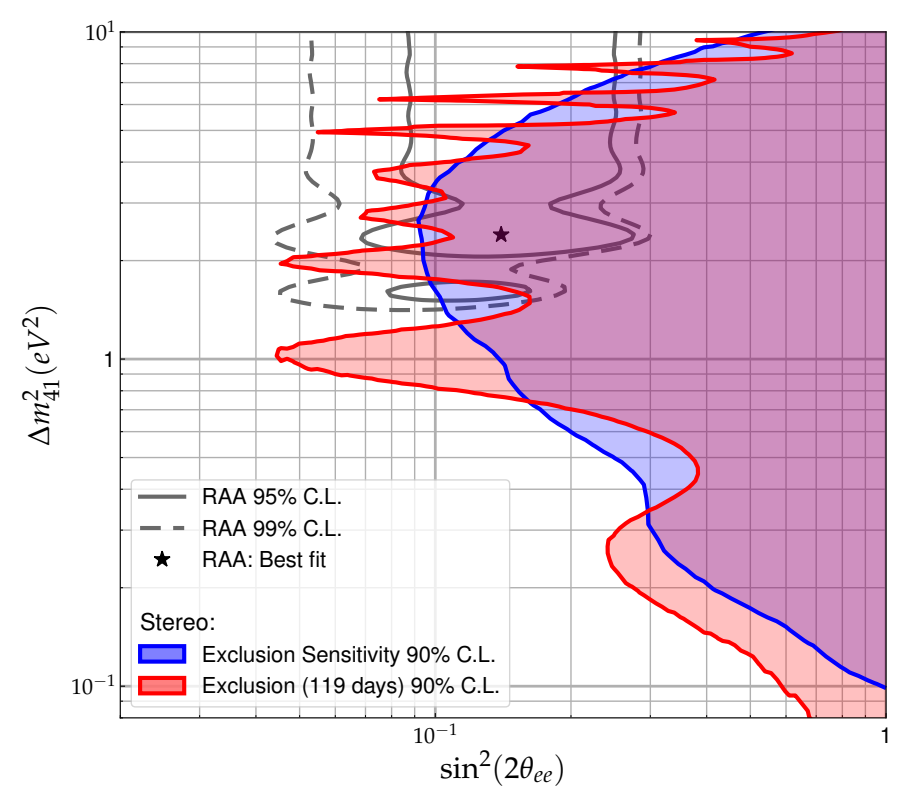

Figure 5: Exclusion contour at $90 \%$ C.L. (red). The original RAA contours and best-fit points are indicated with gray lines, and black star, respectively. The expected sensitivity is shown in blue.

\section{References}

[1] G. Mention et al, Phys. Rev. D 83, 073006 (2011) - DOI: 10.1103/PhysRevD.83.073006

[2] N. Allemandou et al, JINST 121, 16 (2018) - DOI: 10.1088/1748-0221/13/07/P07009

[3] H. Almazán et al, Eur. Phys. J. A. 55, 183 (2019)- DOI: 10.1140/epja/i2019-12886-y

[4] H. Almazán et al, Phys. Rev. Lett. 13, 07 (2018) - DOI: 10.1103/PhysRevLett.121.161801 